\title{
Immune responses in patients with esophageal cancer treated with SART1 peptide-pulsed dendritic cell vaccine
}

\author{
MIWAKO NARITA $^{1}$, TATSUO KANDA ${ }^{2}$, TAKASHI ABE ${ }^{3}$, TAKAYOSHI UCHIYAMA ${ }^{1}$, \\ MINAMI IWAFUCHI ${ }^{1}$, ZHIYIN ZHENG ${ }^{3}$, AICHUN LIU $^{3}$, TSUTOMU KAIFU $^{2}$, SHINICHI KOSUGI ${ }^{2}$, \\ MASAHIRO MINAGAWA ${ }^{2}$, KYOGO ITOH $^{4}$ and MASUHIRO TAKAHASHI ${ }^{1}$ \\ ${ }^{1}$ Laboratory of Hematology and Oncology, Graduate School of Health Sciences, Niigata University, \\ Niigata 951-8518; Departments of ${ }^{2}$ Surgery and ${ }^{3}$ Hematology, Endocrinology, and Metabolism, \\ School of Medicine, Niigata University, Niigata 951-8520; ${ }^{4}$ Cancer Vaccine Development Division, \\ Kurume University Research Center for Innovative Cancer Therapy, Fukuoka 830-0011, Japan
}

Received October 3, 2014; Accepted November 20, 2014

DOI: 10.3892/ijo.2015.2846

\begin{abstract}
Patients with advanced stage of squamous cell carcinoma of esophagus have a poor prognosis with a lethal outcome. In order to explore the feasibility and effectiveness of dendritic cell (DC)-based immunotherapy for squamous cell carcinoma of esophagus, we performed a phase I/II clinical trial of monocyte-derived dendritic cells (moDCs) pulsed with SART1 peptide in seven patients with advanced stage of this disease. Although the feasibility of this therapy was definite, the effectiveness was not clearly confirmed in advanced stage of squamous cell carcinoma of esophagus. However, in vitro study revealed that moDCs generated for this therapy possessed a potent ability of inducing SART1 peptide-specific cytotoxic T lymphocytes (CTLs). In addition, these moDCs were demonstrated to be able to produce exosomes with an antigen presenting ability for inducing SART1 peptide-specific CTLs. ELISPOT assay using cryopreserved patient's lymphocytes demonstrated that IFN- $\gamma$ ELISPOTs were increased after four times of SART1 peptide-pulsed moDC vaccinations compared with before the vaccination in a patient. The present study demonstrated that moDCs prepared from advanced stage of squamous cell carcinoma of esophagus possess a good immune function and in vivo immune responses (detected by ELISPOT assay) were evoked by the infusion of these moDCs. These findings suggest that DC-based immunotherapy could be one of the modalities applicable for squamous cell carcinoma of esophagus.
\end{abstract}

Correspondence to: Dr Miwako Narita, Laboratory of Hematology and Oncology, Graduate School of Health Sciences, Niigata University, 2-746 Asahimachi-dori, Chuo-ku, Niigata 951-8518, Japan

E-mail: naritami@clg.niigata-u.ac.jp

Key words: dendritic cells, esophageal cancer, SART1, antigenspecific CTL, exosomes, ELISPOT

\section{Introduction}

In Japan, $>90 \%$ of esophageal cancer is squamous cell carcinoma, which prognosis is poorer than adenocarcinoma of esophagus (1). Patients with advanced stage of esophageal cancer have a poor prognosis with a lethal outcome, despite efforts to improve diagnostic procedures and treatment modalities (2).

Dendritic cells (DCs), which are potent antigen-presenting cells, could coordinate innate and adaptive immune responses. Hence, DC-based tumor immunotherapy was introduced in the patients with prostate cancer, melanoma, renal cell carcinoma, glioma, gastric cancer, colon cancer, and pediatric solid tumor (3). In 2010, monocyte-derived dendritic cells (moDCs) pulsed with fusion antigen protein consisting of full-length prostatic acid phosphatase (PAP) and full-length GM-CSF were approved by U.S. Food and Drug Administration for the treatment of men with hormone refractory prostate cancer. Cellular immunotherapy using these moDCs prolonged overall survival among men enrolled in the phase III clinical study compared with placebo group (4). As demonstrated in phase III trials of this prostate cancer immunotherapy, moDC-based cellular immunotherapy was shown to be effective in cancer patients when the candidate is selected properly. However, with regard to DC-based cellular immunotherapy for carcinoma of esophagus, only few studies (5-7) have been carried out. One of the studies dealt with WT1 peptide-pulsed DC therapy with activated $\mathrm{T}$ lymphocyte therapy for advanced cancers including two patients with esophageal cancer (5). The study showed that there is beneficial effect to some extent. The other two reports dealt with DC therapy for primary malignant melanoma of the esophagus (6,7). On this note, Asakage et al showed that tumor lysate-pulsed DC therapy is a safe and promising approach as adjuvant therapy for primary malignant melanoma of the esophagus (6). Likewise, Ueda et al reported that peptide-specific immune response could be induced in patients with primary malignant melanoma of the esophagus after immunotherapy using DCs pulsed with MAGE peptides (7). 
We performed phase I/II clinical trial of moDCs pulsed with SART1 (8) peptide for patients with advanced squamous cell carcinoma of esophagus. In addition, we performed in vitro studies concerning cytotoxicity of patient's lymphocytes cultured with SART1 peptide-pulsed moDCs or exosomes secreted from these moDCs against esophageal carcinoma cell line. We also performed an IFN- $\gamma$ ELISPOT assay using patient lymphocytes obtained before and after SART1 peptide-pulsed moDC vaccination. Although clinical benefit was not clearly demonstrated, in vitro and in vivo immune responses caused by SART1 peptide-pulsed moDC vaccination was revealed in the present study.

\section{Materials and methods}

Study design. The study was carried out according to a protocol approved by the Institutional Review Board of Niigata University School of Medicine and conducted in accordance with the Helsinki Declaration. Written informed consent was obtained from all patients. Seven patients were enrolled in this open-labeled, non-randomized phase I/II clinical trial. The primary aim of the study was to evaluate feasibility and safety, whereas the secondary aim was to evaluate immunological and clinical responses to DC vaccination (Table I).

Patients and treatment. Eligibility criteria for the present study were as follows: i) advanced or relapsed squamous cell carcinoma of esophagus, which had been treated with standard therapy; ii) presence of HLA-A*24:02; and iii) performance status (PS) $\leq 1$ (ECOG-scale).

The buffy coat cells of patients were collected by leukapheresis (in 14 out of 21 times DC preparation) or bag method (in seven times) with written informed concent. Peripheral blood mononuclear cells (PBMCs) were separated by Ficoll-Hypaque (Lymphoprep; Axis-Shield Poc AS, Oslo, Norway) density centrifugation. Monocytes were isolated by culturing PBMNCs in plastic culture dish (BD Biosciences, San Jose, CA, USA) at a cell concentration of $3-5 \times 10^{6} / \mathrm{ml}$ and removing non-adherent cells from the dish. Immature moDCs were induced from monocytes by culturing plastic adherent cells in the same culture dish containing RPMI-1640 (Invitrogen Life Technologies, Carlsbad, CA, USA) with $5 \%$ autologous serum, $100 \mathrm{ng} / \mathrm{ml} \mathrm{GM-CSF}$ (Kirin Brewery Co., Ltd., Gunma, Japan) and $10 \mathrm{ng} / \mathrm{ml}$ IL-4 (Schering-Plough Research Institute, Kenilworth, NJ, USA) for 7 days. Immature moDCs were matured by adding $10 \mathrm{ng} / \mathrm{ml}$ TNF- $\alpha$ (PeproTech, Inc., Rocky Hill, NJ, USA) on day 6. Mature moDCs were collected from culture dish by pipetting and occasionally using cell scraper (Corning Life Sciences, Tewksbury, MA, USA).

Tumor antigen peptide used for the study was SART1 $_{690-698}$ (EYRGFTQDF, HLA-A*24:02 restricted, GMP grade), which was produced by Multiple Peptide Systems (San Diego, CA, USA) and donated by Prof. Kyogo Itoh (Kurume University, Fukuoka, Japan). SART1 peptide was added to the moDC culture at a concentration of $50 \mu \mathrm{g} / \mathrm{ml}$ during the last $24 \mathrm{~h}$. In the last three patients, keyhole limpet hemocyanin (KLH: carrier protein for peptide antigen) (Calbiochem, La Jolla, CA, USA) was pulsed at a concentration of $50 \mu \mathrm{g} / \mathrm{ml}$ together with SART1 peptide for the last $24 \mathrm{~h}$ of moDC culture (Table I). On occasion, PBMNCs and peptide-pulsed moDCs were cryopreserved for later in vitro study.

On the day of vaccination, peptide-pulsed moDCs were washed and re-suspended in $500 \mu 1$ saline with 5\% autologous serum and transferred to a $1 \mathrm{ml}$ syringe for injection. The moDC suspension was injected intravenously (IV) in the first four patients (patients 1-4) and subcutaneously (SC) in the upper arm in the last three patients (patients 5-7). Infusions with peptide-pulsed moDCs were repeated every three weeks up to five times depending on the patient.

Clinical evaluation. Evaluation with CT scan and clinical examinations were performed before, during and after vaccinations. A skin test for delayed-type hypersensitivity (DTH) reaction was performed using an intradermal injection of $100 \mu \mathrm{l}$ of the peptide or KLH (5 mg/ml each) on the palmar side of the forearm. Saline solution $(100 \mu \mathrm{l})$ was used as a negative control. More than $2 \mathrm{~mm}$ red induration area after $48 \mathrm{~h}$ was defined as a positive DTH skin test reaction.

Analysis of surface phenotypes of moDCs prepared for infusion. Immediately before injection, antigen-pulsed moDCs were spared and subjected to phenotypic analysis as previously described (9). The cells were stained by incubation with monoclonal antibodies against CD1a (Immunotech, Marseille, France), CD14, CD80, CD86 and HLA-DR (BD Biosciences) together with the relevant isotype controls to analyze the expression of cell surface antigens.

Preparation of moDC-derived exosomes. moDC-derived exosomes were prepared using the method described by Zitvogel et al (10). The whole culture medium of SART1-pulsed moDCs was harvested at the time of preparing moDCs for injection. moDC culture medium was centrifuged at $300 \mathrm{xg}$ for $20 \mathrm{~min}$ and the supernatant was collected. The supernatant was centrifuged at $10,000 \mathrm{x}$ g for $30 \mathrm{~min}$ and the supernatant was collected again for eliminating cell debris. Then the supernatant was ultra-centrifuged at $100,000 \mathrm{x} \mathrm{g}$ for $60 \mathrm{~min}$ and the pellet was collected and washed once in a large volume of medium. Exosome pellet was finally dissolved at the concentration of exosomes derived from $10^{7}$ moDCs in $1 \mathrm{ml}$ RPMI-1640 medium. The protein concentration in the exosome preparation was measured and used for SART1 peptide-specific cytotoxic T lymphocyte (CTL) induction assay.

Identification of exosome. Exosome was identified by demonstrating the expressions of both HLA-DR and CD86 on the surface of the nanoscale vesicle (Fig. 1) (11). Anti-PE microbeads (Miltenyi Biotec GmbH, Bergisch Gladbach Germany) were incubated with PE labeled anti-HLA-DR monoclonal antibody (BD Biosciences) for $1 \mathrm{~h}$ at $4^{\circ} \mathrm{C}$ with tapping every $10 \mathrm{~min}$. The mixture was washed with PBS by centrifuging at $9,100 \times \mathrm{g}(10,000 \mathrm{rpm})$ for $10 \mathrm{~min}$ at $4^{\circ} \mathrm{C}$ twice in order to eliminate free monoclonal antibody. Microbeads pellet was suspended with Fc receptor blocking solution (PBS with $0.5 \%$ human $\gamma$-globulin and $0.1 \%$ sodium azide) (for blocking the $\mathrm{Fc}$ receptors possibly expressed on the surface of exosome) and mixed with exosome solution, and then incubated for $1.5 \mathrm{~h}$ at $4^{\circ} \mathrm{C}$ with tapping every $20 \mathrm{~min}$. The mixture was washed 


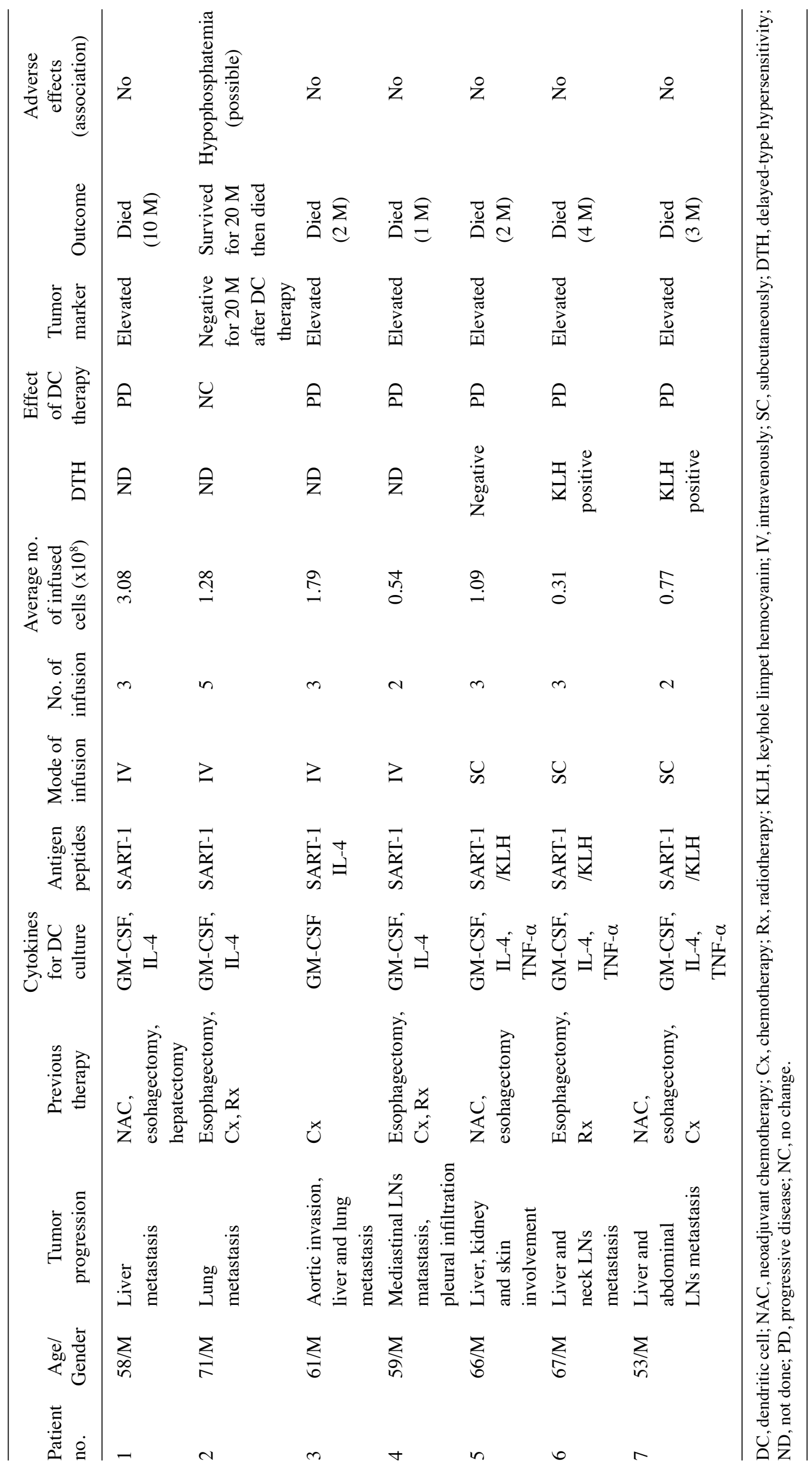




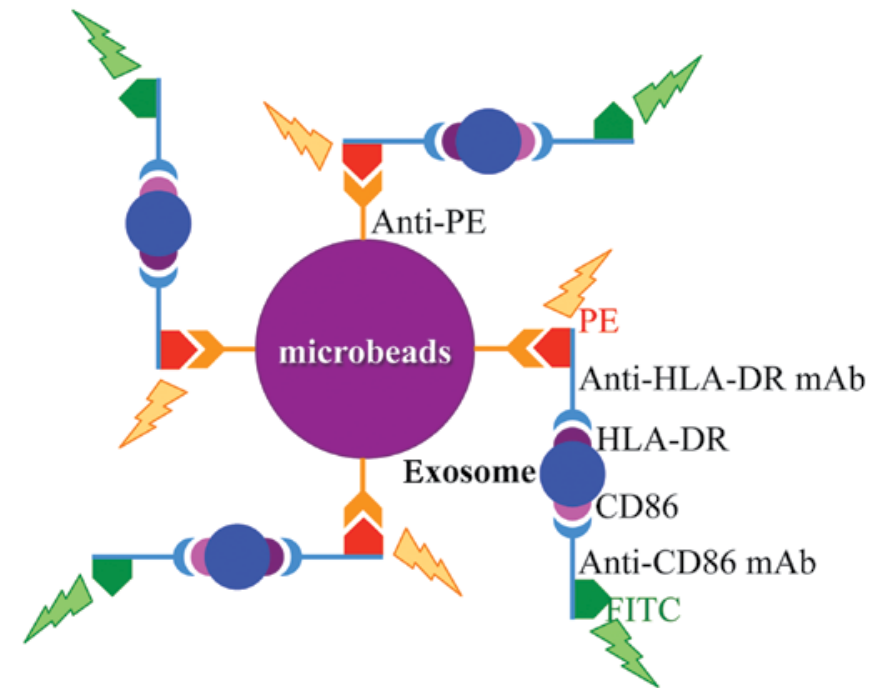

Figure 1. Binding fashion among exosomes, microbead, anti-HLA-DR mAb and anti-CD86 mAb. Exosome was identified by its binding capacity with both anti-HLA-DR mAb (bound with microbead) and anti-CD86 mAb.

with PBS by centrifuging at 9,100 x g (10,000 rpm) for $10 \mathrm{~min}$ at $4^{\circ} \mathrm{C}$ twice in order to eliminate free exosomes. Microbeads bound with exosomes were incubated with FITC-labeled anti-CD86 monoclonal antibody for $1 \mathrm{~h}$ at $4{ }^{\circ} \mathrm{C}$ with tapping every $10 \mathrm{~min}$. The mixture was washed with PBS by centrifuging at $9,100 \mathrm{x} \mathrm{g}(10,000 \mathrm{rpm})$ for $10 \mathrm{~min}$ at $4^{\circ} \mathrm{C}$ twice. Microbeads pellet was suspended with PBS and processed for flow cytometry analysis. Anti-PE microbeads, anti-PE microbeads bound with PE-labeled anti-HLA-DR monoclonal antibody (in excess of microbeads), and anti-FITC microbeads bound with FITC-labeled anti-HLA-DR monoclonal antibody (in excess of microbeads) were used for compensation of flow cytometry analysis. RPMI-1640 medium with 5\% of human serum was used as control for exosomes.

Proliferation assay. Proliferation assay was performed for evaluating an antigen-specific proliferative capacity of lymphocytes in moDC-treated patients. Briefly, SART1 peptide-pulsed moDCs, which were used for vaccination, were irradiated with $30 \mathrm{~Gy}$ of ${ }^{137} \mathrm{Cs}$ generated from gamma irradiation apparatus (PS-3000SB Cs-137; Pony Industry Co., Ltd., Osaka, Japan) immediately before MLC. One hundred thousand allogeneic or patient's autologous PBMCs, which were collected before or after 3rd vaccination then cryopreserved, were co-cultured in a 96-well flat-bottom microtiter plate (BD Biosciences) with graded numbers of moDCs. Co-cultured cells were pulsed with $0.5 \mu \mathrm{Ci}(18.5 \mathrm{kBq}) /$ well $\left[\right.$ methyl- ${ }^{3} \mathrm{H}$ ]-thymidine (PerkinElmer, Boston, MA, USA) on day 5 of culture and harvested after overnight culture with a cell harvester (Labo Mash; Futaba Medical Inc., Tokyo, Japan). Cellular proliferation was evaluated by measuring ${ }^{3} \mathrm{H}$-thymidine incorporation with a liquid scintillation counter (LSC-5100; Aloka Co., Ltd., Tokyo, Japan). The experiments were performed in triplicate.

SART1 peptide-specific CTL induction using peptide-pulsed moDCs. Patient's PBMCs were co-cultured with SART1 peptide-pulsed and irradiated moDCs at a cell ratio of $10: 1$ in 24 -well plate containing $2 \mathrm{ml}$ of $5 \%$ autologous serum-containing RPMI-1640 medium as described previously (12). IL-2 (Shionogi \& Co., Ltd., Osaka, Japan) and IL-7 (Cytheris S.A., Vanves, France) were added to the co-culture on day 3 at the final concentration of $50 \mathrm{U} / \mathrm{ml}$ and $10 \mathrm{ng} / \mathrm{ml}$, respectively. Two thirds of the medium with IL-2 and -7 were replenished every 2-3 days throughout the culture period. Patient's MNCs were stimulated repeatedly every week with the same cryopreserved and thawed peptide-pulsed moDCs and the co-culture was maintained for 4 weeks. For CTL induction by exosomes derived from SART1-pulsed moDCs, autologous PBMCs were cultured in $2 \mathrm{ml}$ autologous serum-containing medium with $500 \mu \mathrm{l}$ of exosome solution, which contains exosomes derived from $5 \times 10^{6}$ moDCs. Addition of IL-2 and -7, and replenishment with fresh medium were undertaken in the same manner as the co-culturing with SART1 peptide-pulsed moDCs.

Cytotoxicity assay. Patient lymphocytes, which were cultured with SART1 peptide-pulsed autologous moDCs or their exosomes for 4 weeks, were used as effector cells in ${ }^{51} \mathrm{Cr}$-release cytotoxicity assay by the method described previously (9). Esophageal cancer cell line, KE4 cells (expressing of HLA-A*24 and SART1), and chronic myelogenous leukemia-blastic crisis (CML-BC) cell line, C2F8 cells (expressing HLA-A*24 but not SART1) (13) were used as target cells for the cytotoxicity assay. Target cells $\left(1 \times 10^{6}\right)$, were labeled with $100 \mu \mathrm{Ci}(100 \mu \mathrm{l})$ of $\mathrm{Na}^{51} \mathrm{CrO}_{4}$ (NEN Life Sciences Inc., Boston, MA, USA) and cultured with effector cells in a 96-well round bottom plate (BD Biosciences) at $37^{\circ} \mathrm{C}$ in a fully humidified $5 \% \mathrm{CO}_{2}$ atmosphere. Cytotoxicity of effector cells was determined at various effector-target cell ratios after incubation for $4 \mathrm{~h}$. The supernatants of the co-culture were then harvested and analyzed for ${ }^{51} \mathrm{Cr}$ release in an auto-well gamma system ARC-300 (Aloka Co., Ltd.). Maximum and spontaneous ${ }^{51} \mathrm{Cr}$ release was measured after incubation of labeled target cells with $1 \mathrm{~N}$ HCL or medium alone, respectively. Percentages of cytotoxicity of the effector cells were calculated using the following formula: \% cytotoxicity $=\left[\left({ }^{51} \mathrm{Cr}\right.\right.$ release of sample wells - spontaneous ${ }^{51} \mathrm{Cr}$ release)/(maximum ${ }^{51} \mathrm{Cr}$ release - spontaneous ${ }^{51} \mathrm{Cr}$ release)] x100.

ELISPOT assay. Cryopreserved patients' PB-MNCs were plated in $2 \mathrm{ml} /$ well at a concentration of $2 \times 10^{6}$ cells in 24-well plates (BD Biosciences) in 5\% human serum-containing RPMI-1640 medium with $10 \mu \mathrm{g} / \mathrm{ml}$ of SART1 peptide. Two days later, $300 \mathrm{IU} / \mathrm{ml}$ IL-2 was added to the cultures. The cultured cells were tested for reactivity in the ELISPOT on day 12. The ELISPOT assay for quantifying SART1 peptide-specific IFN- $\gamma$-releasing cells was performed using ELISpot ${ }^{\text {PLUS }}$ for Human IFN- $\gamma$ kit (Mabtech AB, Nacka Strand, Sweden). The cultured cells and SART1 peptides were added to the ELISPOT plates, which had been coated with anti-IFN- $\gamma$ antibody (1-D1K), and the plates were incubated overnight. The following day, biotinylated detection antibody (7-B6-1-biotin) was added to the washed wells. The plates were incubated for $2 \mathrm{~h}$ and washed, and the streptavidin-ALP was added to each well. Plates were incubated at room temperature for $1 \mathrm{~h}$, and the enzyme substrate 5-bromo-4-chloro-3-indolyl phosphate/nitroblue tetrazolium (BCIP/NBT-plus) was added to each well and incubated at room temperature for $15 \mathrm{~min}$. 
The spots were counted using stereomicroscope imaging system (Olympus Corp., Tokyo, Japan), and the peptide-specific CTL frequency could be calculated from the numbers of spot-forming cells.

Statistical analysis. The statistical relevance of differences in ${ }^{3} \mathrm{H}$-thymidine incorporation in proliferation assay and ${ }^{51} \mathrm{Cr}$ release in cytotoxicity assay was evaluated with a two-way ANOVA, applying GraphPad Prism Software (GraphPad Software, Inc., San Diego, CA, USA). Differences were considered as significant at $\mathrm{p}<0.05$ and markedly significant at $\mathrm{p}<0.01$.

\section{Results}

Patient characteristics and vaccination with peptide-pulsed moDCs. Seven patients with advanced stage of squamous cell carcinoma of esophagus were enrolled for the study. Patient characteristics are shown in Table I. The mean age of the patients was 62 years (range, 53-71 years) and all were males. All the patients, who had developed metastases to liver, lung or kidney and so on, were treated with operation, chemotherapy and/or radiotherapy before entering the clinical trial. In the first four patients, moDCs were prepared by culturing monocytes with GM-CSF/IL-4 and pulsing with SART1 peptide. In the last three patients, TNF- $\alpha$ was used for maturation of moDCs generated by culturing monocytes with GM-CSF/IL-4 and mature moDCs were pulsed with KLH in combination with SART1. Infusions of moDCs were undertaken two to five times, IV or SC in the first four patients, and in the last three patients, respectively.

The average number of infused cells in each infusion varied $\left(0.31-3.08 \times 10^{8}\right.$ cells) from patient to patient depending on the method of blood drawing. Pre-culture number of MNCs, number of all infused cells, and percentage of large cells estimated by forward scatter and side scatter dot plots of flow cytometry, as well as the number of DCs (large cells) in each infusion of individual patient are shown in Table II. Comparison of these values among leukapheresis and bag method for blood drawing is shown in Table III. Mean and SD of moDCs in a single infusion procedure was $0.37 \pm 0.32 \times 10^{8}$ cells in whole with $0.50 \pm 0.31 \times 10^{8}$ cells in leukapheresis and $0.12 \pm 0.09 \times 10^{8}$ cells in bag method (Table III).

Surface phenotypes and allogeneic antigen presenting abilities of injected moDCs. In the first four patients, immature moDCs were generated by culture with GM-CSF and IL-4 and pulsed with SART1 peptide only. In these patients, immature moDCs were used for the therapy since immature DCs were presumed to mature physiologically in the process of interaction with $\mathrm{T}$ cells in vivo. In the last three patients, mature moDCs were generated by culture with TNF- $\alpha$ in addition to GM-CSF and IL-4 and pulsed with SART1 peptide and KLH. These prepared moDCs were analyzed for surface phenotypes relating to antigen presentation. Although moDCs prepared from all the enrolled patients were positive for CD1a, CD80 CD86 and HLA-DR, the expression of CD83 was much higher in moDCs generated by culture with TNF- $\alpha$ in addition to GM-CSF and IL-4 compared with those generated by culture with GM-CSF and IL-4 only (Fig. 2). Therefore, the

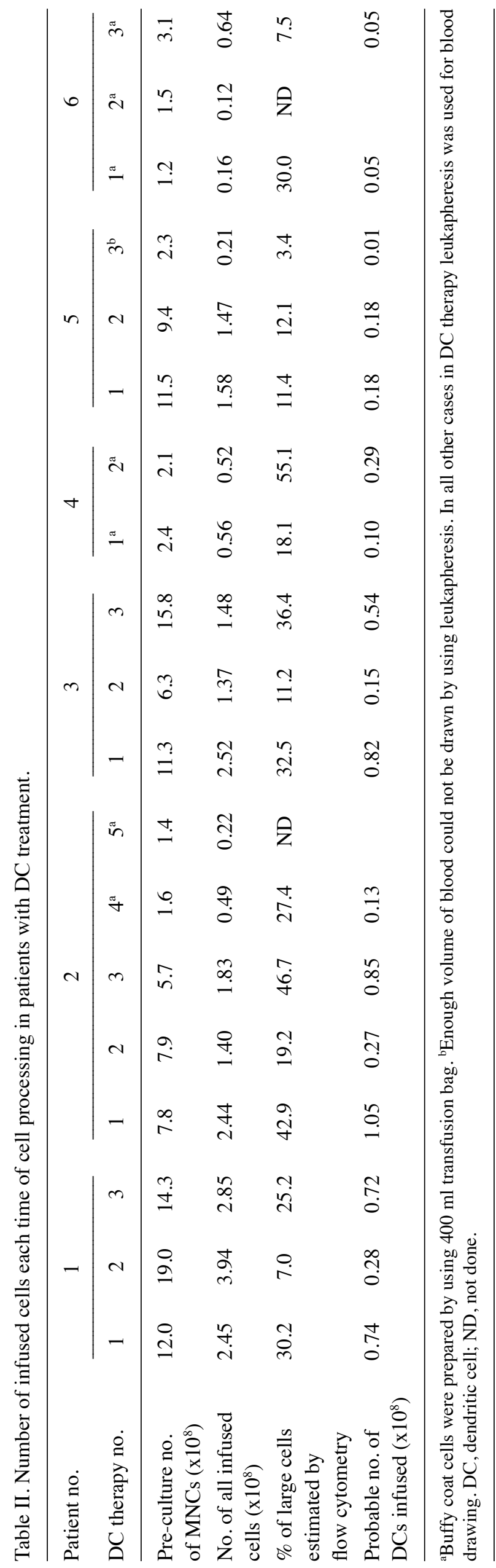


Table III. Comparison of number (mean \pm SD) of infused cells among leukapheresis and the bag method.

\begin{tabular}{|c|c|c|c|c|}
\hline Blood drawing & All $(n=21)$ & Leukapheresis $(\mathrm{n}=13)$ & $\operatorname{Bag}(n=7)$ & $\begin{array}{c}\text { Insufficient } \\
\text { leukapheresis }(n=1)\end{array}$ \\
\hline $\begin{array}{l}\text { Pre-culture no. of } \\
\operatorname{MNCs}\left(\times 10^{8}\right)\end{array}$ & $7.42 \pm 5.19$ & $10.78 \pm 3.68$ & $1.90 \pm 0.62$ & 2.30 \\
\hline $\begin{array}{l}\text { No. of all infused } \\
\text { cells }\left(\times 10^{8}\right)\end{array}$ & $1.32 \pm 1.02$ & $1.91 \pm 0.86$ & $0.39 \pm 0.20$ & 0.21 \\
\hline $\begin{array}{l}\% \text { of large cells estimated } \\
\text { by flow cytometry }\end{array}$ & $24.9 \pm 14.36$ & $25.6 \pm 12.8$ & $27.6 \pm 15.9$ & 3.40 \\
\hline $\begin{array}{l}\text { Probable no. of DCs } \\
\text { infused }\left(\times 10^{8}\right)\end{array}$ & $0.37 \pm 0.32$ & $0.50 \pm 0.31$ & $0.12 \pm 0.09$ & 0.01 \\
\hline
\end{tabular}

DC, dendritic cell.

\section{Patient}

no.

CD1a
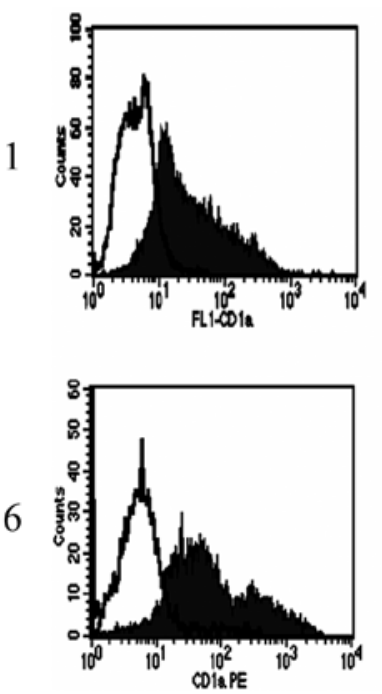

CD80
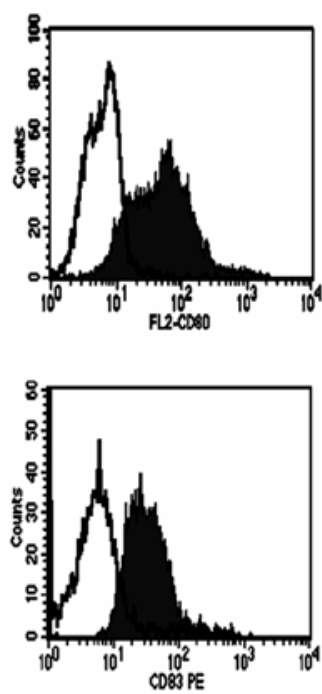

CD83
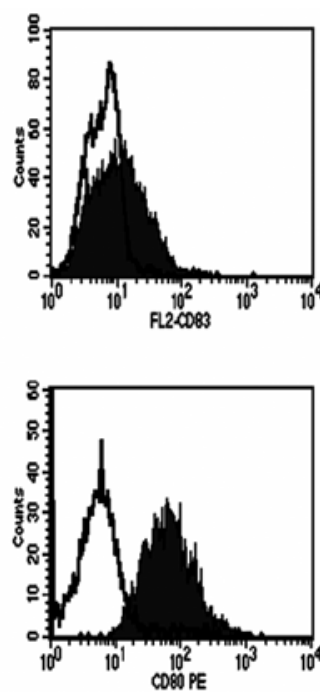

CD86
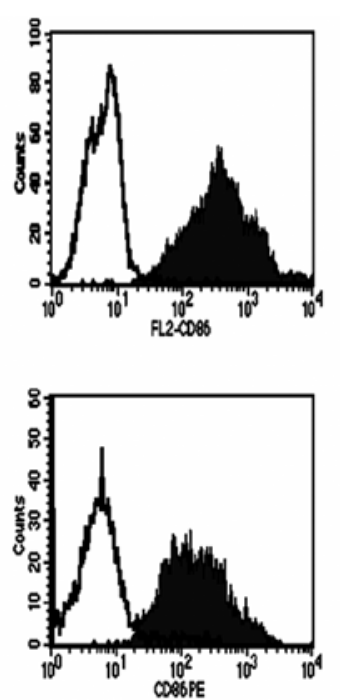

HLA-DR
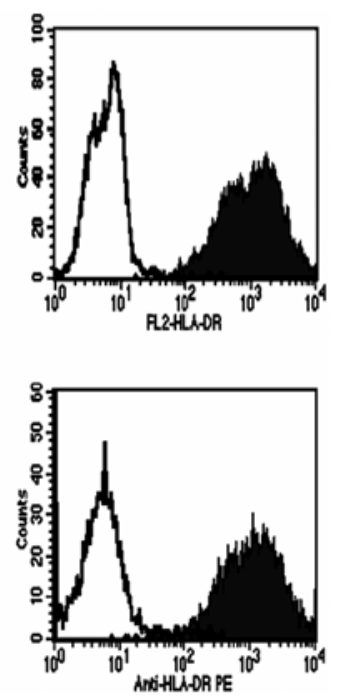

Figure 2. Surface phenotypes of monocyte-derived dendritic cells (moDCs) prepared for dendritic cell (DC)-based immunotherapy. Flow cytometry analysis was performed for surface phenotypes of moDCs generated from PB adherent cells of patient no. 1 and 6 . Culture with GM-CSF/IL-4 for 7 days was used for generating moDCs in patient no. 1 and TNF- $\alpha$ was added for the last $24 \mathrm{~h}$ to GM-CSF/IL-4-induced moDCs in patient no. 6 . These moDCs were pulsed with SART1 peptide or SART1 peptide plus keyhole limpet hemocyanin (KLH) during the last $24 \mathrm{~h}$ before analysis in patient no. 1 or 6 , respectively. Black line shows a histogram with control IgG1 and the filled histogram reveals surface expression of each molecule analyzed using monoclonal antibodies depicted above the histograms. Histograms of moDCs from patient no. 1 or 6 were similar to those of moDCs generated in patient no. 1-4 or 5-7, respectively.

surface phenotypes of infused moDCs were characteristic for immature moDCs in the first four patients and mature moDCs in the last three patients.

These moDCs were analyzed for antigen presenting ability by using allogeneic proliferation assay. Although mature moDCs showed slightly higher ${ }^{3} \mathrm{H}$-thymidine incorporation than immature moDCs in low stimulator/responder ratio of the proliferation assay, immature and mature moDCs were demonstrated to possess a considerable potent ability of antigen presentation (Fig. 3).

DTH and effects of DC therapy. Although skin DTH reactions against KLH were detected in two out of three patients vaccinated with moDCs pulsed with SART1 and KLH, DTH reaction against SART1 peptide was not observed in all the seven patients (Table I). One patient who received SART1 peptide-pulsed moDC vaccine (patient no. 2) remained stable for 20 months after moDC therapy judging from tumor marker and CT findings and he was categorized as no change (NC). But thereafter he developed lung metastasis, for which the operation was undertaken. The remaining six patients had progressive disease (PD) with the median survival of 3.7 months and no favorable response was observed during and after the vaccination course (Table I).

Toxicity. The vaccination was generally well-tolerated and no allergic reaction to the vaccine was observed. One patient who received SART1 peptide-pulsed moDCs showed a moderate 

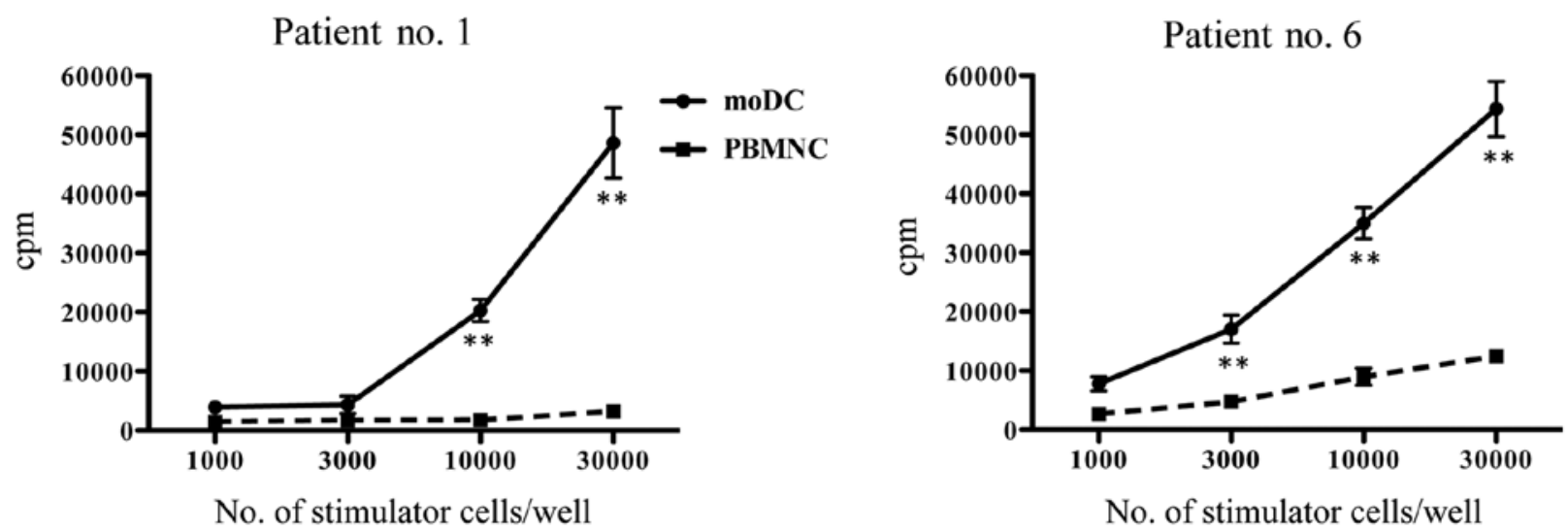

Figure 3. Allogeneic antigen presenting ability of monocyte-derived dendritic cells (moDCs) prepared for DC-based immunotherapy. ${ }^{3} \mathrm{H}$-thymidine incorporation in proliferation assay performed by using moDCs generated from patient no. 1 or 6 . Culture with GM-CSF/IL-4 for 7 days was used for generating moDCs in patient no. 1 and TNF- $\alpha$ was added for the last $24 \mathrm{~h}$ to GM-CSF/IL-4-induced moDCs in patient no. 6. These moDCs were pulsed with SART1 peptide or SART1 peptide plus keyhole limpet hemocyanin $(\mathrm{KLH})$ during the last $24 \mathrm{~h}$ before the assay in patient no. 1 or 6 , respectively. Red line shows cpm of ${ }^{3} \mathrm{H}$-thymidine incorporated by the stimulation with prepared moDCs and blue line by the stimulation with third party PBMNCs. ${ }^{3} \mathrm{H}$-thymidine incorporations in patient no. 1 or 6 was similar to those obtained by using moDCs generated in patient nos. 1-4 or 5-7, respectively. ${ }^{* *}$ Markedly significant differences (p<0.01) between moDCs and PBMNCs.

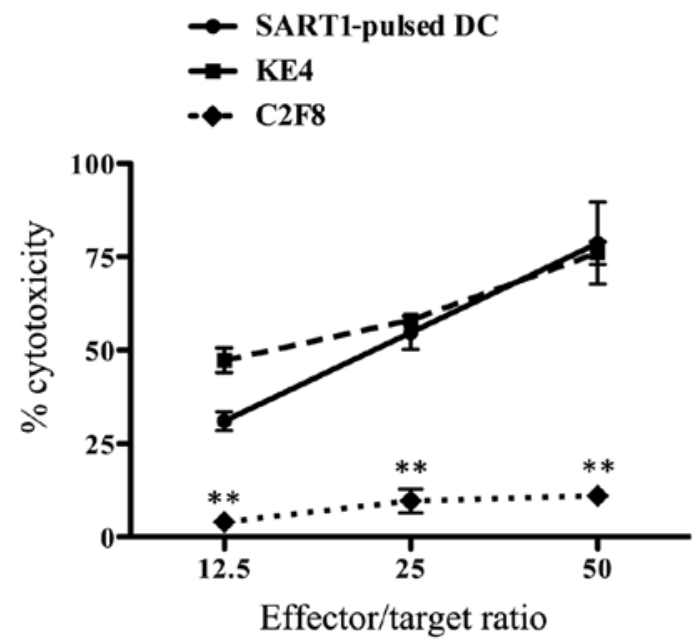

Figure 4. Induction of SART1-specific cytotoxic T lymphocytes (CTLs) using SART1 peptide-pulsed monocyte-derived dendritic cells (moDCs) PBMNCs of patient no. 7, which had been primed in vitro with autologous SART1 peptide/keyhole limpet hemocyanin (KLH)-pulsed moDCs three times, were analyzed for their cytotoxicity against SART1 peptide-pulsed autologous moDCs, KE4 cells (esophageal cancer cell line with expression of both HLA-A24 and SART1) and C2F8 cells [chronic myelogenous leukemia-blastic crisis (CML-BC) cell line with no expression of SART1]. Cytotoxicity of CTLs was evaluated by percent release of ${ }^{51} \mathrm{Cr}$ from target cells. ${ }^{* *}$ Markedly significant differences $(\mathrm{p}<0.01)$ between C2F8 and SART1 peptide-pulsed moDCs or KE4.

hypophosphatemia, although the relationship with moDC vaccination was not definite (Table I).

Induction of SART1-specific CTLs by using SART1 peptidepulsed moDCs. Lymphocytes of patient no. 7 primed with autologous SART1 peptide/KLH-pulsed moDCs three times showed a significant cytotoxic ability against SART1 peptide-pulsed moDCs and KE4 cells, which were positive for the expression of both HLA-A24 and SART1, in an effector-to-target ratio dependent manner. However, CML-BC cell line C2F8 cells (13), which did not express SART1,

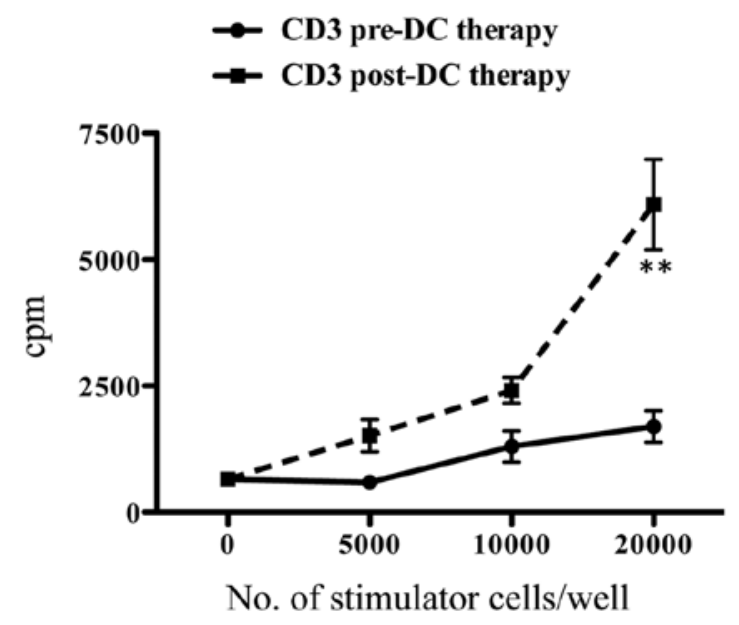

Figure 5. Increased reactivity of vaccinated patient lymphocytes against SART1 peptide/keyhole limpet hemocyanin (KLH)-pulsed monocyte-derived dendritic cells (moDCs). Reactivity of $\mathrm{CD}^{+} \mathrm{T}$ cells of patient no. 6 prepared at pre-treatment phase and those at post-vaccination phase (after three times therapy of SART1 peptide/KLH-pulsed moDCs) was investigated by performing proliferation assay using moDCs pulsed with SART1 peptide/KLH as stimulator cells. ${ }^{* *}$ Markedly significant differences $(\mathrm{p}<0.01)$ between $\mathrm{CD}^{+} \mathrm{T}$ cells at pre-dendritic cell (DC) therapy phase and $\mathrm{CD}^{+}$ $\mathrm{T}$ cells at post-DC therapy phase.

were not killed by lymphocytes primed with SART1/KLH peptide-pulsed moDCs (Fig. 4).

Increased reactivity of vaccinated patient's lymphocytes against SART1 peptide/KLH-pulsed moDCs. Reactivity of $\mathrm{CD}^{+} \mathrm{T}$ cells of patient no. 6 against moDCs pulsed with SART1 peptide/KLH was compared between $\mathrm{CD}^{+} \mathrm{T}$ cells in pre-treatment phase and those in post-vaccination phase (after three times infusion of SART1 peptide/KLH-pulsed moDCs). $\mathrm{CD}^{+} \mathrm{T}$ cells in post-vaccination phase showed a much higher reactivity against SART1 peptide/KLH-pulsed moDCs in autologous MLC compared with those in pre-treatment phase (Fig. 5). This enhancement of $\mathrm{CD}^{+} \mathrm{T}$-cell reactivity 

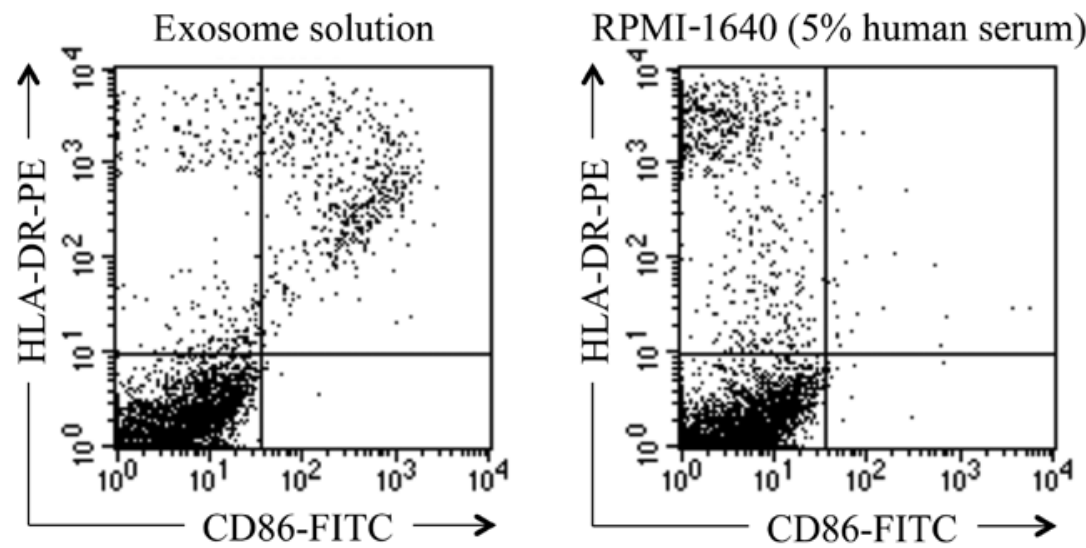

Figure 6. Identification of exosomes in ultra-centrifuged preparation of moDC supernatant. Anti-PE microbeads, which were bound with PE-labeled anti-HLA-DR monoclonal antibody, were incubated with exosome solution, and then stained with FITC-labeled anti-CD86 monoclonal antibody. Microbeads were analyzed for double staining by flow cytometry. RPMI-1640 medium with 5\% human serum was used as control for exosomes.

A

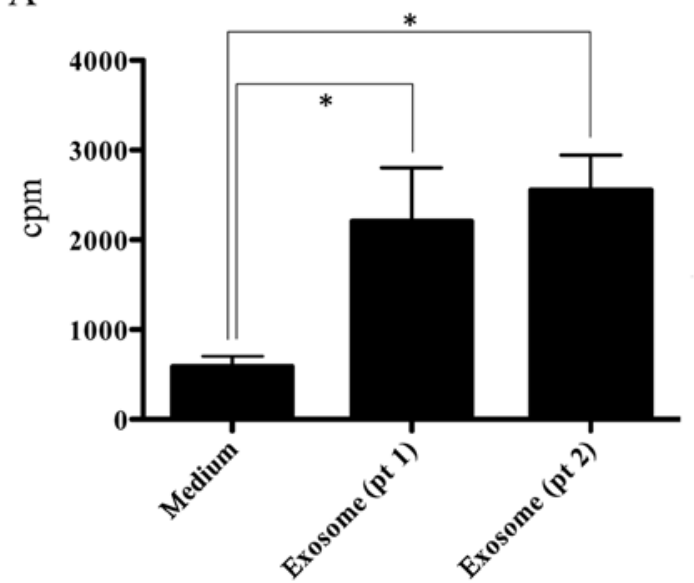

B

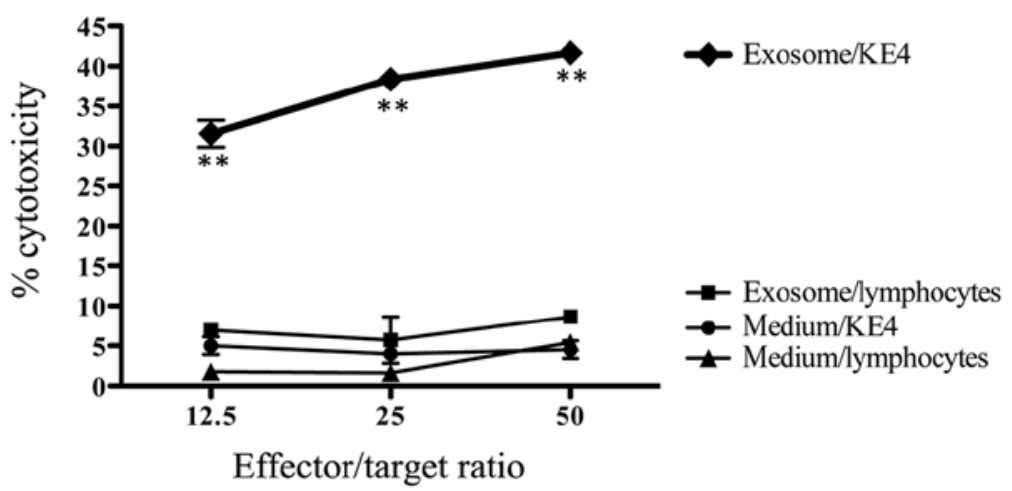

Figure 7. Production of antigen-presenting exosomes by monocyte-derived dendritic cells (moDCs). Exosome pellet was prepared by ultra-centrifugation of supernatant from SART1-pulsed moDC culture in patient no. 1 and 2. Exosome pellet was dissolved at the concentration of exosomes derived from $10^{7}$ moDCs in $1 \mathrm{ml}$ RPMI-1640 medium. (A) For evaluating an allogeneic antigen-presenting ability of exosome solution, 100,000 allogeneic peripheral blood mononuclear cells (PBMCs) were cultured in $200 \mu \mathrm{l}$ FBS-containing medium with $50 \mu 1$ of exosome solution, which is equivalent to exosomes derived from $5 \times 10^{5}$ moDCs. (B) For evaluating an antigen-specific cytotoxic T lymphocyte (CTL)-inducting ability of these exosomes, autologous PBMCs were cultured in $2 \mathrm{ml}$ autologous serum-containing medium with $500 \mu \mathrm{l}$ of exosome solution, which is equivalent to exosomes derived from $5 \times 10^{6}$ moDCs. PBMCs cultured with exosomes for 4 weeks were used as effector cells in ${ }^{51} \mathrm{Cr}$-release cytotoxicity assay using KE4 cells (HLA-A*24 $/ \mathrm{SART1}^{+}$) and allogeneic lymphocytes as target cells. "Significant differences $(\mathrm{p}<0.05)$ between medium and exosome solutions from patient no. 1 or $2 .{ }^{* *}$ Markedly significant differences $(\mathrm{p}<0.01)$ between exosome against KE4 and medium against KE4, medium against lymphocytes or exosome against lymphocytes.

was thought to be mainly caused by an increased reactivity against KLH.

Production of antigen-presenting exosomes by moDCs. Ultra-centrifuged preparation from moDC supernatant of patient no. 1 was demonstrated to possess microvesicles expressing both HLA-DR and CD86, which were presumed to be exosomes. However,HLA-DR-bound microbeads were negative for CD86 in RPMI-1640 with 5\% human serum (Fig. 6). Exosome solutions prepared from moDC cultures of patient no. 1 and 2 were used as substitute for stimulator cells in MLC using allogeneic PBMNCs as responder cells. Exosome solutions from both patients were demonstrated to possess a weak but definite antigen presenting ability to allogeneic lymphocytes (Fig. 7A). Exosome solution prepared from patient no. 1 was shown to induce SART1-specific CTLs in 4 weeks-culture of autologous PBMNCs when stimulated three times with exosome solution derived from moDCs (Fig. 7B).

SART1-specific T-cell response by ELISPOT. PBMNCs obtained from three patients (patient no. 2,3 and 4) before moDC vaccination and at time points during the vaccinations were analyzed for quantifying SART1 peptide-specific IFN- $\gamma$-releasing cells. One (patient no. 2) of three patients had a SART1-specific immune response in ELISPOT assay of lymphocytes at day 84 from the initiation of the vaccination (after four times of moDC vaccination) (Fig. 8). In the other two patients (patient nos. 3 and 4), SART1-specific IFN- $\gamma$-releasing cells did not increase probably due to the short period after the vaccination (not $>42$ days from the initiation of moDC vaccination). Patient no. 2 , who showed a definite increase of IFN- $\gamma$ ELISPOT after moDC vaccination, is the 


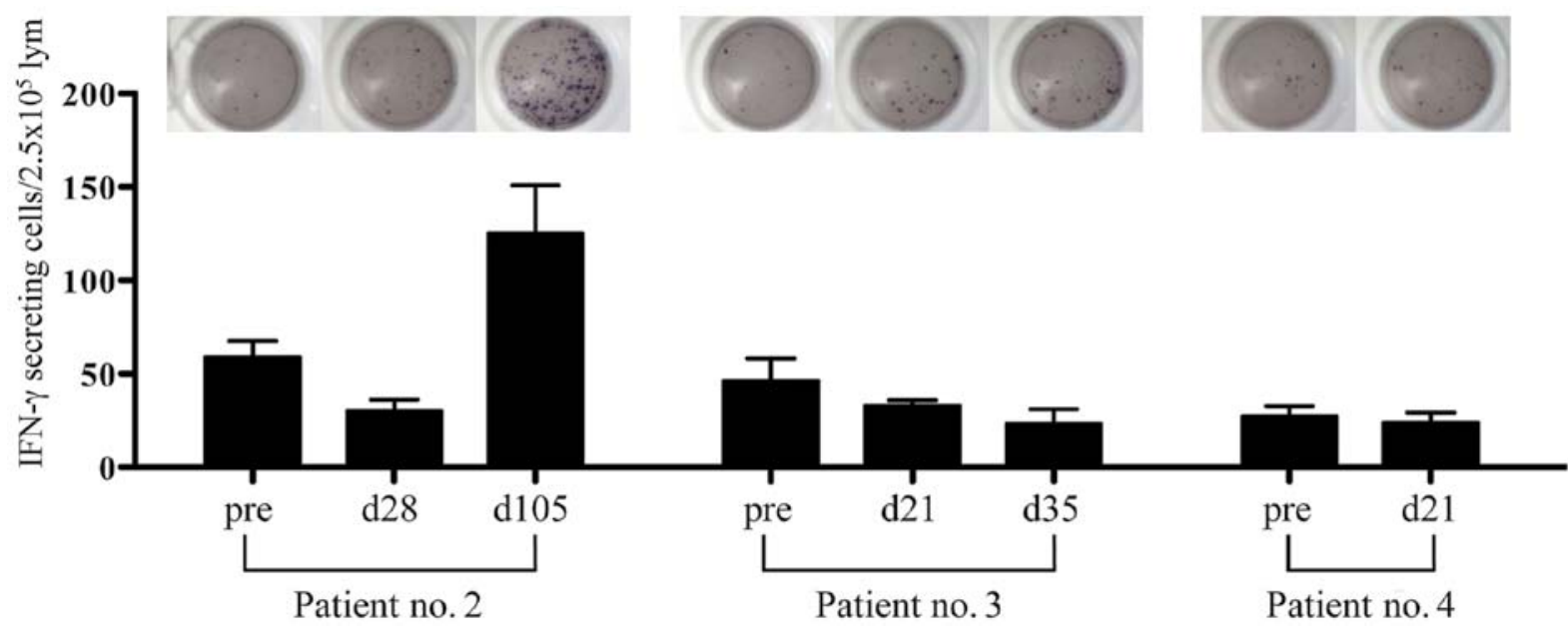

Figure 8. Enumeration of SART1-specific T cells by ELISPOT assay in three patients (patient nos. 2, 3 and 4). Lymphocytes before monocyte-derived dendritic cell (moDC) vaccination and at time points during the vaccinations were cryopreserved for the study. The thawed cells were cultured with SART1 peptide and IL-2 for 12 days for the first step culture, then the cultured cells were washed and placed with SART1 peptide in the wells of ELISPOT plate for the second step culture. Numbers of responding T cells against SART1 peptide before moDC vaccination and at time points during the vaccinations are the mean \pm SEM of triplicate experiments

case identified to be stable disease after infusion of SART1 peptide-pulsed moDCs.

\section{Discussion}

DC-based antitumor immunotherapy has been demonstrated to be feasible without side-effects and bring clinical benefits with immune responses (14), but esophageal cancers have been rarely enrolled in DC therapy so far. There have been several in vitro studies indicating DC immunotherapy as a promising strategy for esophageal cancer. Milano and Krishnadath reported a patient-specific autologous readout assay for pre-clinical testing of DC-mediated cytotoxic immune responses. They demonstrated that the use of DCs transfected with autologous total tumor RNA could be effective for treating esophageal cancer (16). While in the migration study of administered DCs, Fujiwara et al performed an intratumoral administration of in-labeled DCs in combination with preoperative chemotherapy in esophageal cancer patients. Their study revealed that the intratumoral administration of DCs during chemotherapy does not give rise to DC migration from the tumor to the draining lymph nodes, and suggested that an impairment of DC migration may be associated with difficulty in achieving an optimal clinical response in DC therapy (15). It is now generally recognized that clinical outcomes of patients receiving DC vaccination alone for advanced stage cancer have not been satisfactory. Therefore, the treatment strategy to combine DC therapy with another treatment modality to improve clinical outcomes is considered (16). With regard to antigen peptide-based immunotherapy for esophageal squamous cell carcinoma, Kono et al reported that the immune response induced by multiple-peptides vaccination could make the prognosis better by analyzing the data of a multicenter phase II clinical trial consisting of 60 patients with advanced stage of esophageal cancer (17).

Exosomes, which are nanoscale (50-100 $\mathrm{nm}$ ) vesicles, can mediate an immune response by activating $\mathrm{T}$ lymphocytes (through antigen presentation), natural killer (NK) cells (through NKG2D ligand binding), and DCs (through antigen transfer) (18). Exosomes secreted by DCs loaded with tumor antigen have been shown to generate potent immune responses against cancer cells by inducing antigen-specific $\mathrm{CD}^{+} \mathrm{T}$ cells (19) and abolishing the suppressive function of regulatory $\mathrm{T}$ cells (20). Until now, only three clinical trials have been undertaken, on the application of exosomes for antitumor immunotherapy. Dai et al reported that autologous ascites-derived exosomes combined with GM-CSF could induce tumor antigen-specific CTL responses in phase I clinical trial for patients with colorectal cancer, with no to minimal adverse effects (21). Escudier et al disclosed that phase I clinical trial of autologous DC derived-exosomes was feasible and safe in patients with melanoma and minor to stable clinical responses were observed in skin and lymph node sites (22). Furthermore, Morse et al demonstrated a MAGE-specific T-cell response and increased NK lytic activity in patients with non-small cell lung carcinoma treated with autologous DC derived-exosomes loaded with multiple MAGE peptides (23).

Safety and efficacy were explored in the current phase I/II vaccination for patients with esophageal cancer. The vaccination was well-tolerated and no side-effect except for possible hypophosphatemia was observed, similar to those reported in other vaccination studies (24-26). One patient (patient no. 2) treated with SART1-pulsed moDCs remained stable for 20 months after moDC therapy, although thereafter he developed lung metastasis, for which surgery was undertaken. In patient no. 2 , we could observe that the number of IFN- $\gamma$-producing cells increased after four times of SART1-pulsed moDC vaccination by IFN- $\gamma$ ELISPOT assay. The other six patients died after 1-10 months from vaccination with PD. Although clinical and survival benefits were not observed in this vaccination treatment for the enrolled patients with advanced stage of squamous cell carcinoma of esophagus, feasibility of tumor antigen peptide-pulsed moDC therapy was demonstrated. In the present clinical trial, DTH against antigen peptide was negative, although positive for $\mathrm{KLH}$ in some patients. We used peptide itself for priming 
in DTH. Instead of antigen peptides, antigen peptide-pulsed DCs should have been used for priming in DTH. On this note, Ellebaek et al reported that antigen-pulsed DCs should be used as antigen in DTH test in order to present antigens to obtain the highest local immune reactivity (27). Also in vitro, the reactivity of patient's $\mathrm{CD} 3^{+} \mathrm{T}$ cells against SART1 peptide/KLH-pulsed moDCs increased after three times DC vaccination. This enhancement of $\mathrm{CD}^{+} \mathrm{T}$-cell reactivity was presumed to be due to an increased reactivity against KLH but not against SART1 peptide as shown in vivo of DTH. On the contrary, moDCs prepared from each patient expressed molecules associated with antigen presentation, such as CD1a, CD80, CD83, CD86 and HLA-DR, although the expression of CD83 among them was influenced by the culture method with or without TNF- $\alpha$. Patient's lymphocytes primed with SART1 peptide-pulsed moDCs were demonstrated to have a significant cytotoxic ability against HLA-A $24^{+} / \mathrm{SART}^{+}$ esophageal carcinoma cell line and SART1 peptide-pulsed autologous moDCs. These SART1 peptide-pulsed moDCs prepared from enrolled cancer patients were shown to produce antigen-presenting exosomes, which could generate SART1-specific CTLs in culture of autologous lymphocytes being stimulated with exosome preparation. In addition, ELISPOT assay using cryopreserved lymphocytes of the patients demonstrated that IFN- $\gamma$ ELISPOTs were increased after four times of moDC vaccinations in one patient. These findings suggest that injected moDCs had an ability to induce antigen-specific CTLs and the patient lymphocytes acquired antigen-specific reactivity when primed with antigens presented by injected moDCs. In the present clinical application of antigen peptide-pulsed moDCs for advanced stage of squamous cell carcinoma of esophagus and related in vitro and in vivo studies, it was shown that DC-based cellular immunotherapy for these cancer patients was feasible, functional DCs could be generated from these patients, and patient's immunity is elevated by the infusion of DCs prepared from monocytes. In order to establish a clinically effective DC-based immunotherapy, the patient indication criteria for these therapies and the manner of preparing highly qualified DCs for injection were presumed to be the principle issues.

\section{References}

1. Siewert JR and Ott K: Are squamous and adenocarcinomas of the esophagus the same disease? Semin Radiat Oncol 17: 38-44, 2007.

2. Lagergren J and Lagergren P: Oesophageal cancer. BMJ 341: c6280, 2010

3. Palucka $\mathrm{K}$ and Banchereau J: Cancer immunotherapy via dendritic cells. Nat Rev Cancer 12: 265-277, 2012.

4. Kantoff PW, Higano CS, Shore ND, Berger ER, Small EJ, Penson DF, Redfern CH, Ferrari AC, Dreicer R, Sims RB $\mathrm{Xu}$ Y, Frohlich MW and Schellhammer PF; IMPACT Study Investigators: Sipuleucel-T immunotherapy for castration-resistant prostate cancer. N Engl J Med 363: 411-422, 2010.

5. Kato Y: WT1 peptide pulsed dendritic cell therapy with activated T lymphocytes therapy for advanced cancers. Gan To Kagaku Ryoho 37: 2240-2242, 2010 (In Japanese).

6. Asakage M, Kitayama J, Tsuno NH, Komuro Y, Kaisaki S, Hori N, Nagawa H, Tsuno NH, Hori N and Takahashi K: Primary malignant melanoma of the esophagus treated by esophagectomy and adjuvant dendritic-cell therapy. J Gastroenterol 40: 545-546, 2005.
7. Ueda Y, Shimizu K, Itoh T, Fuji N, Naito K, Shiozaki A, Yamamoto Y, Shimizu T, Iwamoto A, Tamai H and Yamagishi H: Induction of peptide-specific immune response in patients with primary malignant melanoma of the esophagus after immunotherapy using dendritic cells pulsed with MAGE peptides. Jpn J Clin Oncol 37: 140-145, 2007.

8. Kikuchi M, Nakao M, Inoue Y, Matsunaga K, Shichijo S, Yamana $\mathrm{H}$ and Itoh K: Identification of a SART-1-derived peptide capable of inducing HLA-A24-restricted and tumor-specific cytotoxic T lymphocytes. Int J Cancer 81: 459-466, 1999.

9. Narita M, Tochiki N, Saitoh A, Watanabe N, Kaji M, Satoh N, Yamahira A, Nakamura T, Masuko M, Furukawa T, Toba K, Fuse I, Aizawa Y and Takahashi M: Induction of antigen-specific cytotoxic T lymphocytes by using monocyte-derived DCs transfected with in vitro-transcribed WT1 or SART1 mRNA. Med Oncol 26: 429-436, 2009.

10. Zitvogel L, Regnault A, Lozier A, Wolfers J, Flament C, Tenza D, Ricciardi-Castagnoli P, Raposo G and Amigorena S: Eradication of established murine tumors using a novel cell-free vaccine: dendritic cell-derived exosomes. Nat Med 4: 594-600, 1998.

11. Théry C, Boussac M, Véron P, Ricciardi-Castagnoli P, Raposo G, Garin $\mathbf{J}$ and Amigorena S: Proteomic analysis of dendritic cell-derived exosomes: a secreted subcellular compartment distinct from apoptotic vesicles. J Immunol 166: 7309-7318, 2001.

12. Yamahira A, Narita M, Nakamura T, Watanabe N, Kaji M, Taniguchi T, Hashimoto S, Furukawa T, Toba K, Aizawa Y, Kuzushima K and Takahashi M: Generation of antigen-specific cytotoxic T lymphocytes using a leukemic plasmacytoid dendritic cell line as antigen presenting cells. Leuk Res 35: 793-799, 2011.

13. Furukawa T, Koike T, Ying W, Kishi K, Aoki S, Gotoh T, Hashimoto S, Saitoh H, Hanano M, Shinada S, et al: Establishment of a new cell line with the characteristics of a multipotential progenitor from a patient with chronic myelogenous leukemia in early erythroblastic crisis. Leukemia 8: 171-180, 1994.

14. Shore ND, Mantz CA, Dosoretz DE, Fernandez E, Myslicki FA, McCoy C, Finkelstein SE and Fishman MN: Building on sipuleucel-T for immunologic treatment of castration-resistant prostate cancer. Cancer Control 20: 7-16, 2013.

15. Fujiwara S, WadaH,MiyataH,KawadaJ,Kawabata R,NishikawaH, Gnjatic S, Sedrak C, Sato E, Nakamura Y, Sakakibara M, Kanto T, Shimosegawa E, Hatazawa J, Takahashi T, Kurokawa Y, Yamasaki M, Nakajima K, Takiguchi S, Nakayama E, Mori M and Doki Y: Clinical trial of the intratumoral administration of labeled DC combined with systemic chemotherapy for esophageal cancer. J Immunother 35: 513-521, 2012.

16. Milano F and Krishnadath KK: Novel therapeutic strategies for treating esophageal adenocarcinoma: the potential of dendritic cell immunotherapy and combinatorial regimens. Hum Immunol 69: 614-624, 2008.

17. Kono K, Iinuma H, Akutsu Y, Tanaka H, Hayashi N, Uchikado Y, Noguchi T, Fujii H, Okinaka K, Fukushima R, Matsubara H, Ohira M, Baba H, Natsugoe S, Kitano S, Takeda K, Yoshida K, Tsunoda T and Nakamura Y: Multicenter, phase II clinical trial of cancer vaccination for advanced esophageal cancer with three peptides derived from novel cancer-testis antigens. J Transl Med 10: 141, 2012.

18. Théry C, Ostrowski M and Segura E: Membrane vesicles as conveyors of immune responses. Nat Rev Immunol 9: 581-593, 2009.

19. Hao S, Bai O, Li F, Yuan J, Laferte S and Xiang J: Mature dendritic cells pulsed with exosomes stimulate efficient cytotoxic T-lymphocyte responses and antitumour immunity. Immunology 120: 90-102, 2007.

20. Taieb J, Chaput N, Schartz N, Roux S, Novault S, Ménard C, Ghiringhelli F, Terme M, Carpentier AF, Darrasse-Jèze G, Lemonnier F and Zitvogel L: Chemoimmunotherapy of tumors: cyclophosphamide synergizes with exosome based vaccines. J Immunol 176: 2722-2729, 2006.

21. Dai S, Wei D, Wu Z, Zhou X, Wei X, Huang H and Li G: Phase I clinical trial of autologous ascites-derived exosomes combined with GM-CSF for colorectal cancer. Mol Ther 16: 782-790, 2008.

22. Escudier B, Dorval T, Chaput N, André F, Caby MP, Novault S, Flament C, Leboulaire C, Borg C, Amigorena S, Boccaccio C, Bonnerot C, Dhellin O, Movassagh M, Piperno S, Robert C, Serra V, Valente N, Le Pecq JB, Spatz A, Lantz O, Tursz T, Angevin $\mathrm{E}$ and Zitvogel L: Vaccination of metastatic melanoma patients with autologous dendritic cell (DC) derived-exosomes: results of the first phase I clinical trial. J Transl Med 3: 10, 2005. 
23. Morse MA, Garst J, Osada T, Khan S, Hobeika A, Clay TM Valente N, Shreeniwas R, Sutton MA, Delcayre A, Hsu DH, Le Pecq JB and Lyerly HK: A phase I study of dexosome immunotherapy in patients with advanced non-small cell lung cancer. J Transl Med 3: 9, 2005.

24. Berntsen A, Trepiakas R, Wenandy L, Geertsen PF, thor Straten P, Andersen MH, Pedersen AE, Claesson MH, Lorentzen T, Johansen JS and Svane IM: Therapeutic dendritic cell vaccination of patients with metastatic renal cell carcinoma: a clinical phase 1/2 trial. J Immunother 31: 771-780, 2008.

25. Trepiakas R, Berntsen A, Hadrup SR, Bjørn J, Geertsen PF, Straten PT, Andersen MH, Pedersen AE, Soleimani A, Lorentzen T, Johansen JS and Svane IM: Vaccination with autologous dendritic cells pulsed with multiple tumor antigens for treatment of patients with malignant melanoma: results from a phase I/II trial. Cytotherapy 12: 721-734, 2010.
26. Svane IM, Pedersen AE, Johnsen HE, Nielsen D, Kamby C, Gaarsdal E, Nikolajsen K, Buus S and Claesson MH: Vaccination with p53-peptide-pulsed dendritic cells, of patients with advanced breast cancer: report from a phase I study. Cancer Immunol Immunother 53: 633-641, 2004.

27. Ellebaek E, Engell-Noerregaard L, Iversen TZ, Froesig TM, Munir S, Hadrup SR, Andersen MH and Svane IM: Metastatic melanoma patients treated with dendritic cell vaccination, Interleukin-2 and metronomic cyclophosphamide: results from a phase II trial. Cancer Immunol Immunother 61: 1791-1804, 2012. 